\title{
Detection of weak stochastic forces in a parametrically stabilized micro-optomechanical system
}

\author{
A. Pontin, ${ }^{1,2}$ M. Bonaldi, ${ }^{3,1}$ A. Borrielli, ${ }^{3,1}$ F. S. Cataliotti, ${ }^{4,5,6}$ F. Marino, ${ }^{6,7}$ G. A. Prodi, ${ }^{1,2}$ E. Serra,,${ }^{1,8,9}$ and F. Marin ${ }^{4,5,6, *}$ \\ ${ }^{1}$ Istituto Nazionale di Fisica Nucleare (INFN), Trento Institute for Fundamental Physics and Application, I-38123 Povo, Trento, Italy \\ ${ }^{2}$ Dipartimento di Fisica, Università di Trento, I-38123 Povo, Trento, Italy \\ ${ }^{3}$ Institute of Materials for Electronics and Magnetism, Nanoscience-Trento-FBK Division, 38123 Povo, Trento, Italy \\ ${ }^{4}$ Dipartimento di Fisica e Astronomia, Università di Firenze, Via Sansone 1, I-50019 Sesto Fiorentino (FI), Italy \\ ${ }^{5}$ European Laboratory for Non-Linear Spectroscopy (LENS), Via Carrara 1, I-50019 Sesto Fiorentino (FI), Italy \\ ${ }^{6}$ INFN, Sezione di Firenze, 50019 Sesto Fiorentino (FI), Italy \\ ${ }^{7}$ CNR-INO, L.go Enrico Fermi 6, I-50125 Firenze, Italy \\ ${ }^{8}$ Department of Microelectronics and Computer Engineering/ECTM/DIMES, Delft University of Technology, \\ Feldmanweg 17, 2628 CT Delft, The Netherlands \\ ${ }^{9}$ Interdisciplinary Laboratory for Computational Science (LISC), FBK-University of Trento, I-38123 Povo (TN), Italy
}

(Received 15 January 2014; published 28 February 2014)

\begin{abstract}
Measuring a weak force is an important task for micromechanical systems, both when using devices as sensitive detectors and, particularly, in experiments of quantum mechanics. The optimal strategy for resolving a weak stochastic signal force on a huge background (typically given by thermal noise) is a crucial and debated topic, and the stability of the mechanical resonance is a further, related critical issue. We introduce and analyze the parametric control of the optical spring, which allows us to stabilize the resonance and provides a phase reference for the oscillator motion, yet conserving a free evolution in one quadrature of the phase space. We also study quantitatively the characteristics of our micro-optomechanical system as detector of stochastic force for short measurement times (for quick, high-resolution monitoring) as well as for the longer-term observations that optimize the sensitivity. We compare a simple strategy based on the evaluation of the variance of the displacement which is a widely used technique) with an optimal Wiener-Kolmogorov data analysis. We show that, due to the parametric stabilization of the effective susceptibility, we can more efficiently implement Wiener filtering, and we investigate how this strategy improves the performance of our system. We finally demonstrate the possibility to resolve stochastic force variations well below $1 \%$ of the thermal noise.
\end{abstract}

DOI: 10.1103/PhysRevA.89.023848

PACS number(s): 42.50.Wk, 07.10.Cm, 46.40.Ff, 05.40.Ca

\section{INTRODUCTION}

Micro- and nano-optomechanical systems are the heart of refined force-sensing devices [1-4]. Such systems exploit the huge susceptibility around the resonance of oscillators with excellent mechanical quality factor $Q$, combined with high-sensitivity interferometric measurements. The latter are particularly efficient when the oscillator is embedded in an optical resonator with high optical quality factor, whose optical path depends on the oscillator coordinate. This kind of device is useful both for practical applications and in quantum optics experiments. In both cases, a frequent crucial task is detecting a weak variation of the external force (which we call signal force) on a strong background. For instance, in a quantum experiment, the signal can be due to quantum fluctuations in the radiation pressure, which are usually overwhelmed by background thermal noise (a significant exception is reported in Ref. [4], which presents the first observation of the effect of radiation pressure shot noise on a macroscopic object).

Due to the narrow width of the resonance and, consequently, of the useful sensitive band with respect to typical input force, it is meaningful to discuss the general problem of detecting a weak signal force with flat spectral density (white spectrum) in the presence of a white background force, taking into account a given sensitivity to the oscillator displacement (i.e., a flat readout noise spectrum). This can be performed naively by

\footnotetext{
*marin@fi.infn.it
}

measuring the area of the resonance peak emerging from the displacement noise spectrum (or, equivalently, measuring the variance of the oscillator position after band-pass filtering around the resonance). With this estimator, the rate of improvement of the statistical uncertainty for increasing measurement time $t_{\text {meas }}$ depends on the correlation time $\tau_{c}$ of the oscillator motion, with a relative uncertainty scaling as $\sim \sqrt{\tau_{c} / t_{\text {meas }}}$. It seems therefore useful to decrease $\tau_{c}$, i.e., enhance the damping of the oscillator. However, the fluctuation-dissipation theorem implies that such an operation would increase the spectral density of thermal noise. Improved results can instead be achieved by means of a cold damping, e.g., the optical cooling [5-7], which modifies the effective susceptibility and decreases the correlation time without introducing additional noise sources. This technique does not increase the signalto-noise ratio of input excitations, because it changes the response to both signal and background force in the same way. However, as long as the cold damped peak still emerges from the displacement spectral noise, it allows a faster accumulation of statistically independent data bringing therefore, in a given measurement time, to a smaller final uncertainty in the variance of the oscillator motion.

An important remark is that the correlation time of the signal force is by hypothesis very short; therefore the statistics can in principle be much faster than what allowed by the oscillator motion. In other words, the variance of the displacement is not a very efficient indicator, and more refined data analysis can be profitable. In the case of stationary, white input the optimal approach to the measurement is provided by the 
Wiener-Kolmogorov filtering theory [8,9]. This technique requires the preliminary knowledge of the exact response function to the input force and of the signal-to-noise ratio. While the second requirement can be relaxed with a suboptimal but robust filter using a conservative estimate of the sensitivity [10], the accurate knowledge of the susceptibility is a crucial request. Such knowledge is not trivial for micro-optomechanical systems, where the stability of the resonance is affected by several detrimental effects, e.g., thermal phenomena and relaxations of the mechanical oscillator, and above all by the same interaction with the radiation, both due to photothermal effect and to the optomechanical coupling. These considerations suggest that the direct measurement of the spectral peak area could be the only applicable strategy in several kinds of optomechanical systems, and techniques that reduce the effective coherence time of the oscillator motion, such as cold damping or feedback, represent therefore a way to effectively improve the measurement capabilities of the system [1]. However, it has been remarked that optimal resolution is not really improved in this way [11,12], and that appropriate data filtering can completely replace these hardware techniques even in the case of nonstationary, non-Gaussian input [13]. In spite of these correct remarks, the problem of the instability in the oscillator parameters and dynamics remains practically difficult to face, and the implementation of optimal analysis requires a sophisticated technique of adaptive filtering. The experimental demonstration in Ref. [13] indeed keeps short $(\sim \mathrm{ms})$ measurement times. Therefore, even when willing to apply an efficient data analysis, as well as in several kinds of refined optomechanical experiments, stabilization and feedback techniques acting on the optomechanical system are crucial, and indeed this issue has been recently considered by a few groups $[14,15]$.

In this work we present a micro-optomechanical system that includes a parametric stabilization of the resonance by controlling the optical spring. We have proposed and demonstrated this technique in a recent work [16], where the control allows us to prevent instability in a parametrically modulated optomechanical system, thus yielding strong mechanical squeezing. Here we study the characteristics of our system as a detector of stochastic force for short measurement times (for quick, high-resolution monitoring) as well as for long $t_{\text {meas }}$, thus optimizing the sensitivity. We show that, due to the stabilization of the effective susceptibility, we can more efficiently implement Wiener filtering and investigate how this strategy improves the performance of our system.

The article is organized as follows. In Sec. II we describe the theoretical models for the optomechanical interaction, the parametric control of the oscillator, and the strategies exploitable to measure the stochastic force acting on the oscillator; in Sec. II we describe our experimental setup and the measurements; after the Conclusions, in the Appendix we derive the theoretical expressions for the relative uncertainty and discuss the effect of a cutoff in the measured spectra.

\section{MODEL}

\section{A. Optomechanical interaction}

In this section we recall some basic features of the optomechanical interaction. We consider an optical cavity where the resonance frequency depends on an effective
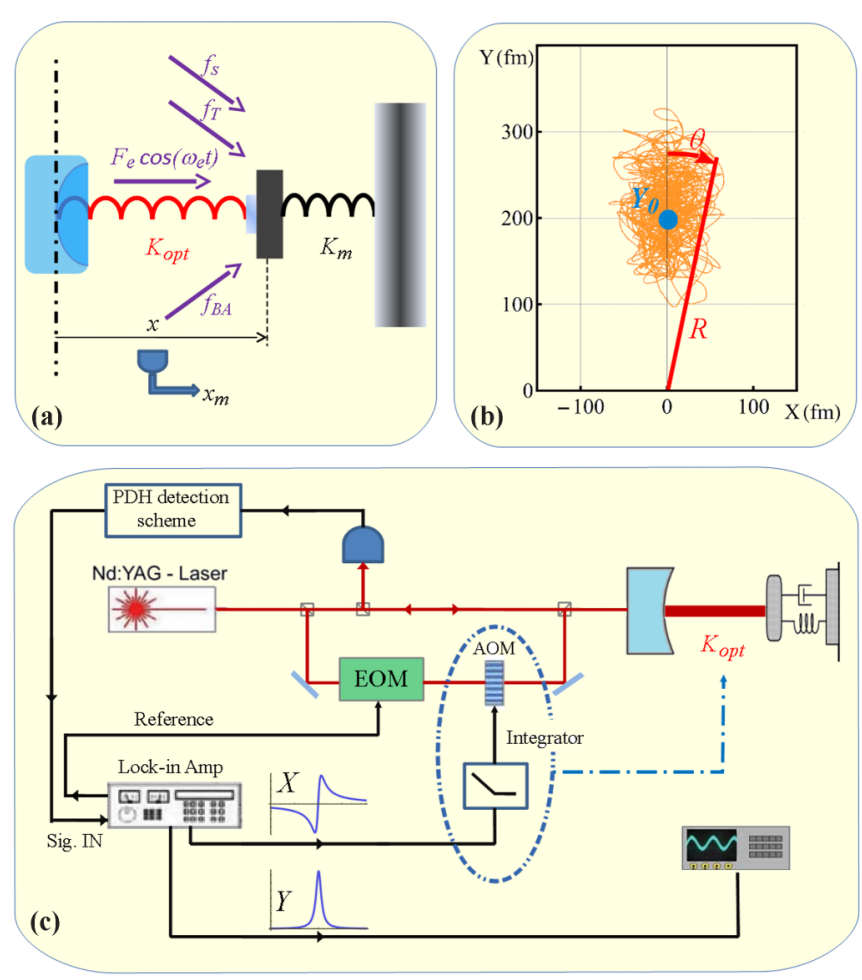

FIG. 1. (Color online) (a) Conceptual scheme of the optomechanical system including measurement and force terms. (b) Experimental measurement of the temporal evolution of the mechanical oscillator in the phase plane, in the configuration with active parametric control. (c) Experimental setup. EOM: electro-optic intensity modulator; dash-dotted lines highlight the parametric control.

coordinate $x$, which is kept at its rest position $x=0$ by elastic forces. The system can be sketched as a linear cavity with a rigid oscillating mirror (Fig. 1) having position $x$, mass $M$, resonance angular frequency $\omega_{\mathrm{m}}$, damping rate $\gamma_{\mathrm{m}}$, and susceptibility $\chi=1 / M\left(\omega_{\mathrm{m}}^{2}-\omega^{2}+i \omega \gamma_{\mathrm{m}}\right)$. The radiation pressure provides a force acting on the mirror, which depends on the detuning $\Delta=\omega_{L}-\omega_{c}$ between the input radiation at frequency $\omega_{L}$ and the cavity resonance at $\omega_{c}$. Since the latter depends on $x$, radiation pressure gives a position-dependent force that can be accounted for by defining an effective susceptibility. Its expression is given by $[7,17]$

$$
\chi_{\mathrm{eff}}(\omega)^{-1}=M\left[\omega_{\mathrm{m}}^{2}-\omega^{2}+i \omega \gamma_{\mathrm{m}}+\frac{|G|^{2} \Delta \omega_{\mathrm{m}}}{(\kappa+i \omega)^{2}+\Delta^{2}}\right]
$$

where $\kappa$ is cavity decay rate and $|G|^{2}$ is the optomechanical coupling, proportional to the intracavity power.

The real part of $\chi_{\text {eff }}$ can be viewed as a combined effect of the mechanical stiffness and an additional spring (optical spring) [18]. The delay in the intracavity field build-up, which gives a contribution to the imaginary part of $\chi_{\text {eff }}$, causes a change in the oscillator damping that allows the optical cooling of its motion [5-7].

For the case of our interest (bad cavity limit $\kappa \gg \omega_{\mathrm{m}}$, small detuning $\Delta \ll \kappa$, and $\omega \approx \omega_{\mathrm{m}}$ ) the expression of optical spring 
constant simplifies to

$$
K_{\mathrm{opt}} \approx-\frac{M|G|^{2} \omega_{\mathrm{m}}}{\kappa^{2}} \Delta
$$

and the optical damping rate to

$$
\gamma_{\mathrm{opt}} \approx \frac{2 K_{\mathrm{opt}}}{M \kappa}
$$

allowing us to write the effective susceptibility as $\chi_{\text {eff }}^{-1}=$ $M\left(\omega_{\text {eff }}^{2}-\omega^{2}+\mathrm{i} \omega \gamma_{\text {eff }}\right)$ with $\gamma_{\text {eff }}=\gamma_{\mathrm{m}}+\gamma_{\mathrm{opt}}$ and

$$
\omega_{\text {eff }}=\sqrt{\omega_{\mathrm{m}}^{2}-K_{\mathrm{opt}} / M} \simeq \omega_{\mathrm{m}}+\frac{|G|^{2}}{2 \kappa^{2}} \Delta .
$$

To our purpose, it is useful to underline that the frequency shift is approximately proportional to the detuning, and therefore a laser beam can be used to control it. Moreover, by varying the working point (detuning) we can choose the effective resonance width $\gamma_{\text {eff }}$ and stabilize it. On the other hand, we remark that in general the optical spring increases the uncertainty and instability of the optomechanical resonance frequency $\omega_{\text {eff }}$ since it is influenced by the noise in the laser intensity (through $G$ ), in the laser frequency and in the cavity length (through $\Delta$ ). In addition, thermal effects due to the absorbed laser power can worsen the intrinsic stability of $\omega_{\mathrm{m}}$.

\section{B. An oscillator with parametric control}

A conceptual scheme of the experiment is shown in Fig. 1(a). We consider an optomechanical oscillator excited by stochastic signal force $f_{s}(t)$ and thermal noise force $f_{T}(t)$ at temperature $T$, with respective spectral densities $S_{s}$ and $S_{T}=2 k_{B} T M \gamma_{\mathrm{m}}$, as well as by a coherent oscillating force of constant amplitude $F_{e} \cos \omega_{\mathrm{e}} t$. The oscillator position $x(t)$ is measured interferometrically by a first laser beam (signal beam). The measurement noise $n(t)$ and the back-action force $f_{B A}(t)$ are considered uncorrelated, with white spectra $S_{n}$ and $S_{B A}$ bounded by

$$
S_{n} S_{B A} \geqslant \hbar^{2} / 4
$$

The evolution of the position $x(t)$ is governed by the stochastic equation

$$
\ddot{x}+\gamma_{\mathrm{eff}} \dot{x}+\omega_{\mathrm{eff}}^{2} x=\frac{1}{M}\left[f_{T}+f_{s}+f_{B A}+F_{e} \cos \omega_{\mathrm{e}} t\right],
$$

and the result of the position measurement is $x_{m}(t)=$ $x(t)+n(t)$.

The motion of the oscillator can be decomposed into two quadratures $X(t)$ and $Y(t)$ in a frame rotating at angular frequency $\omega_{\mathrm{e}}$, according to

$$
x(t)=X(t) \cos \omega_{\mathrm{e}} t+Y(t) \sin \omega_{\mathrm{e}} t .
$$

Assuming $\left|\omega_{\mathrm{e}}-\omega_{\text {eff }}\right| \ll \omega_{\text {eff }}$, and $\gamma_{\text {eff }} \ll \omega_{\text {eff }}$, the evolution equations for the two slowly varying quadratures, derived from Eq. (6), can be written as

$$
\begin{aligned}
\dot{X}+\frac{\gamma_{\mathrm{eff}}}{2} X-\left(\omega_{\mathrm{eff}}-\omega_{\mathrm{e}}\right) Y & =\frac{1}{M \omega_{\mathrm{e}}}\left[f_{T}^{(1)}+f_{s}^{(1)}+f_{B A}^{(1)}\right] \\
\dot{Y}+\frac{\gamma_{\mathrm{eff}}}{2} Y+\left(\omega_{\mathrm{eff}}-\omega_{\mathrm{e}}\right) X & =\frac{1}{M \omega_{\mathrm{e}}}\left[f_{T}^{(2)}+f_{s}^{(2)}+f_{B A}^{(2)}+\frac{F_{e}}{2}\right],
\end{aligned}
$$

where the stochastic force terms have correlation functions $\left\langle f_{a}^{(i)}(t) f_{a}^{(j)}\left(t^{\prime}\right)\right\rangle=\delta_{i j} \delta\left(t-t^{\prime}\right) S_{a} / 2 \quad(i, j=1,2$ and " $a "=$ " $T$," " $s$," " $B A$ "). In the experiment, the two quadratures are measured by sending $x_{m}(t)$ to a lock-in amplifier whose reference signal is derived from the oscillator modulating the coherent force $F_{e}$. The outputs of the lock-in are $X_{m}=$ $X+n^{(1)}$ and $Y_{m}=Y+n^{(2)}$ with $\left\langle n^{(i)}(t) n^{(j)}\left(t^{\prime}\right)\right\rangle=\delta_{i j} \delta(t-$ $\left.t^{\prime}\right) S_{n} / 2$.

The steady-state solutions of Eqs. (8) are the usual components of the oscillator response, as a function of the frequency difference between resonance and excitation $\delta \omega=\omega_{\mathrm{eff}}-\omega_{\mathrm{e}}$ (with steady-state value $\overline{\delta \omega}$ ):

$$
\begin{aligned}
& \bar{X}(\delta \omega)=\frac{F_{e}}{2 M \omega_{e}} \frac{\delta \omega}{\frac{\gamma_{\text {eff }}^{2}}{4}+(\delta \omega)^{2}}, \\
& \bar{Y}(\delta \omega)=\frac{F_{e}}{2 M \omega_{e}} \frac{\gamma_{\mathrm{eff}} / 2}{\frac{\gamma_{\mathrm{eff}}^{2}}{4}+(\delta \omega)^{2}} .
\end{aligned}
$$

We remark that $\bar{X}$ is an odd function of $\overline{\delta \omega}$; therefore it can be efficiently exploited to control and lock $\omega_{\text {eff }}$. The $X_{m}$ quadrature is indeed integrated and sent to control the resonance frequency $\omega_{\text {eff }}$ by modifying the optical spring constant (parametric control). This is obtained in the experiment by acting on the detuning of a second laser beam (control beam) according to

$$
\omega_{L}(t)=\omega_{L}^{0}-\int_{-\infty}^{t} \mathcal{G}\left(t, t^{\prime}\right) X_{m}\left(t^{\prime}\right) d t^{\prime},
$$

where $\omega_{L}^{0}$ is the initial detuning and the kernel $\mathcal{G}\left(t, t^{\prime}\right)$ is constant in the case of an integral feedback loop. Given that $\omega_{L}$ determines the effective frequency $\omega_{\text {eff }}$ via Eq. (4), we can write

$$
\omega_{\mathrm{eff}}(t)=\omega_{\mathrm{eff}}^{0}(t)-\int_{-\infty}^{t} \mathcal{G}_{\mathrm{eff}}\left(t, t^{\prime}\right) X_{m}\left(t^{\prime}\right) d t^{\prime},
$$

where $\omega_{\text {eff }}^{0}(t)$ is the free-running optomechanical frequency and $\mathcal{G}_{\text {eff }} \propto \mathcal{G}$. Equation (3) shows that, in the bad cavity limit, the shift in the resonance frequency $\omega_{\text {eff }}$ due to the optomechanical interaction is larger than the variation in the damping rate $\gamma_{\text {eff }}$; thus the latter can be neglected when considering small variations of $\Delta$ around the working point. We also remark that the control of the optical spring can be considered as a classical effect and its noise neglected in a first-order treatment. In any case, such noise (for us, the radiation pressure noise of the control beam) can be included in $f_{s}$.

At the purpose of analyzing the effect of the control loop, we first consider slow fluctuations in the optomechanical resonance frequency $\omega_{\text {eff }}$, which can be treated as adiabatic changes of the system, keeping the validity of Eqs. (8). In Eq. (11) we replace $X=\bar{X}(\delta \omega)+\delta X$, and, considering small closed-loop fluctuations, we further take $\bar{X}(\delta \omega) \propto \delta \omega$. In the absence of drift in $\omega_{\text {eff }}^{0}(t)$, the steady-state solution is $\delta \omega=0$, i.e., $\omega_{\text {eff }}=\omega_{\mathrm{e}}$ [long term drifts in $\omega_{\text {eff }}^{0}(t)$ can be corrected by additional integrators, as in standard servo-loop systems]. In the phase plane of a reference frame rotating at $\omega_{\mathrm{e}}$, the oscillator motion is now represented by a vector $\mathbf{R}=(X, Y)$ fluctuating around the average value $\left(0, Y_{0}\right)$ with $Y_{0}=\bar{Y}(0)=$ $F_{e} / \gamma_{\text {eff }} M \omega_{e}$ [in Fig. 1(b) we report an experimental example]. 
The feedback loop corrects the fluctuations by counterrotating $\mathbf{R}$ towards the $Y$ axis. If $\mathbf{R}$ remains close to $\left(0, Y_{0}\right)$, i.e., if $\left\langle X^{2}+\left(Y-Y_{0}\right)^{2}\right\rangle \ll Y_{0}^{2}$, we can approximate the angle $\theta$ between $\mathbf{R}$ and the $Y$ axis with $\theta \approx X / Y_{0}$. In this limit, the feedback loop (that acts on $\theta$ ) just influence the fluctuations in the $X$ quadrature, leaving free $Y$ fluctuations. This is expressed by a linear expansion of Eqs. (8) around the steady state, with $\omega_{\mathrm{eff}}=\omega_{\mathrm{e}}+\delta \omega(t), X=\bar{X}+\delta X$, and $Y=Y_{0}+\delta Y$ :

$$
\begin{gathered}
\delta \dot{X}+\frac{\gamma_{\mathrm{eff}}}{2} \delta X-\delta \omega(t) Y_{0}=\frac{1}{M \omega_{\mathrm{e}}}\left[f_{T}^{(1)}+f_{s}^{(1)}+f_{B A}^{(1)}\right] \\
\delta \dot{Y}+\frac{\gamma_{\mathrm{eff}}}{2} \delta Y=\frac{1}{M \omega_{\mathrm{e}}}\left[f_{T}^{(2)}+f_{s}^{(2)}+f_{B A}^{(2)}\right] \\
\delta \omega(t)=\delta \omega_{\mathrm{eff}}^{0}(t)-\int_{-\infty}^{t} \mathcal{G}_{\mathrm{eff}}\left(t, t^{\prime}\right)\left[\bar{X}\left(\delta \omega\left(t^{\prime}\right)\right)+\delta X\left(t^{\prime}\right)\right. \\
\left.+n^{(1)}\left(t^{\prime}\right)\right] d t^{\prime} .
\end{gathered}
$$

We have a few important remarks on the above relations. The first is that the equation governing the fluctuations of the $Y$ quadrature is the same that we would have without feedback; therefore $\delta Y$ behaves as in a free oscillator and, in particular, it can be used to reliably measure the external force. Second, we have a well-defined phase plane: the oscillator is not just frequency stabilized, but also phase locked to the reference. Third, the response function of the $Y$ quadrature is stable, with a peak frequency defined a priori (at $\omega=0$, corresponding to $\omega_{\mathrm{e}}$ for the evolution of $x$ ) and, as a consequence, stable width $\gamma_{\text {eff }}$ and peak signal-to-noise ratio. Such parameters' stability is very important for an easier application of optimal filtering.

The spectrum of the measured $Y_{m}$ quadrature calculated from Eq. (12b) can be written in the form

$$
S_{Y m}=\mathcal{L}(\omega) S_{F}+S_{n} / 2
$$

with

$$
\mathcal{L}(\omega)=\mathcal{A} \frac{\gamma_{\mathrm{eff}}}{\omega^{2}+\left(\frac{\gamma_{\mathrm{eff}}}{2}\right)^{2}},
$$

where $\mathcal{A}=\int_{-\infty}^{\infty} \mathcal{L}(\omega) d \omega / 2 \pi=1 /\left(2 \gamma_{\mathrm{eff}} M^{2} \omega_{\mathrm{e}}^{2}\right)$ and the total force noise spectral density is $S_{F}=S_{S}+S_{T}+S_{B A}$.

The treatment of this section includes slow fluctuations of $\omega_{\text {eff }}^{0}$ as well as fast, although weak, variations that can be considered as phase fluctuations. The case of strong and fast variations of $\omega_{\text {eff }}^{0}$, producing trajectories in the phase plane that take $\mathbf{R}$ far from the region with $\theta<1$, requires a numerical integration of Eqs. (8), and the approximation of a free $Y$ quadrature is no more reliable.

By excluding the coherent excitation and the frequency control, the spectrum of both quadratures, for an optomechanical resonance at $\omega_{\text {eff }}^{0}=\omega_{\mathrm{e}}+\delta \omega$, is

$$
S_{X m}=S_{Y m}=\frac{1}{2}[\mathcal{L}(\omega-\delta \omega)+\mathcal{L}(\omega+\delta \omega)] S_{F}+\frac{S_{n}}{2},
$$

and, in the case of slow fluctuations of $\delta \omega$, the spectral peaks assume the shape of a Voigt profile, maintaining a constant area.

\section{Force measurement strategies}

We consider two possible measurement strategies, with the aim of detecting a weak stochastic signal force $f_{s}$ hidden by the thermal background. In other words, we are seeking for a precise measurement of the stochastic force in order to resolve its weak variations due to changes in $S_{s}$. We are not dealing with measurement accuracy and reproducibility, which both depend critically on absolute calibrations.

The first strategy is simply measuring the area $\sigma^{2}$ of the resonance peak. The advantage of this method is that frequency stability of the optomechanical oscillator is not crucial: the peak area can be calculated by direct integration of the spectrum of $x$ within an appropriate frequency interval, provided that $\omega_{\text {eff }}$ is well within the integration band, and the latter is extended to few $\gamma_{\text {eff }}$ yet maintaining a negligible contribution of the background noise $S_{n}$. The same measurement can be performed, with equal efficiency, on the spectrum of a quadrature. The estimated force spectral density is $E\left\{S_{F}\right\}=\sigma^{2} / \mathcal{A}$. The drawback of this method is the rather slow improvement of the statistical uncertainty, decreasing as $\propto \sqrt{\tau_{c} / t_{\text {meas }}}$ where the correlation time is now $\tau_{c}=1 / \gamma_{\text {eff }}$. The reason is that this strategy does not exploit the full information contained in the signal, whose spectrum around resonance is dominated by the effect of the force fluctuations even well beyond the width $\gamma_{\mathrm{eff}}$.

The second strategy is a close approximation of the Wiener filtering, which represents the optimal choice in case of stationary noise. The noncausal Wiener filter, applied to the spectrum $S_{Y m}$ of Eq. (13), is defined as

$$
|W(\omega)|^{2}=\frac{1}{\mathcal{L}(\omega)}\left[\frac{1}{1+\Gamma \frac{\mathcal{L}(0)}{\mathcal{L}(\omega)}}\right]^{2},
$$

and the maximum information on $S_{F}$ from the experimental $S_{Y m}$ is obtained from the filtered spectrum $S_{W}=|W|^{2} S_{Y m}$. The $1 / \mathcal{L}$ factor in Eq. (16) is a whitening and calibration function, while the term between square brackets is a weight function that requires a preliminary estimate of the noise-to-peak-signal ratio $\Gamma$. Its optimal value is $\Gamma_{\text {opt }}=S_{n} / 2 \mathcal{L}(0) S_{F}$, but an efficient, even if suboptimum, filter can choose a $\Gamma>\Gamma_{\text {opt }}$ [10]. In any case, preliminary fit of a spectrum $S_{Y m}$ allows us to extract the parameters $\gamma_{\text {eff }}$ and $\Gamma$ for the following application of the Wiener filtering procedure. The correlation time of the filtered signal is now $\tau_{c} \sim \sqrt{\Gamma} / \gamma_{\text {eff }}$, yielding a faster improvement of the statistics with $t_{\text {meas }}$ with respect to the previous strategy. For an optimum filter (with $\Gamma=\Gamma_{\text {opt }}$ ), $1 / \tau_{c}$ corresponds to the effective sensitivity bandwidth, i.e., to the frequency band where the effect of force noise falls below the measurement sensitivity [i.e., $\mathcal{L}(\omega) S_{F}=S_{n} / 2$ ]. An example of the application of the whitening function and the complete Wiener filter to a real spectrum is shown in Fig. 2. The force spectral density is estimated by integrating the filtered spectrum $S_{W}$ and dividing the result by the effective bandwidth $\sim 1 / \tau_{c}$. In our real data some spurious peaks appear in the spectrum at few $\mathrm{kHz}$ from the optomechanical resonance; therefore the integration is truncated at $\omega_{\text {cut }} / 2 \pi=3 \mathrm{kHz}$, slightly below $1 / \tau_{c}$. More details on the choice of $\omega_{\text {cut }}$ and on the consequent effective bandwidth are reported in the Appendix.

As we have seen, the application of the Wiener filtering requires the knowledge of the transfer function between force noise and output. For this reason, the parametric control strongly facilitates the filtering procedure, by fixing both the 


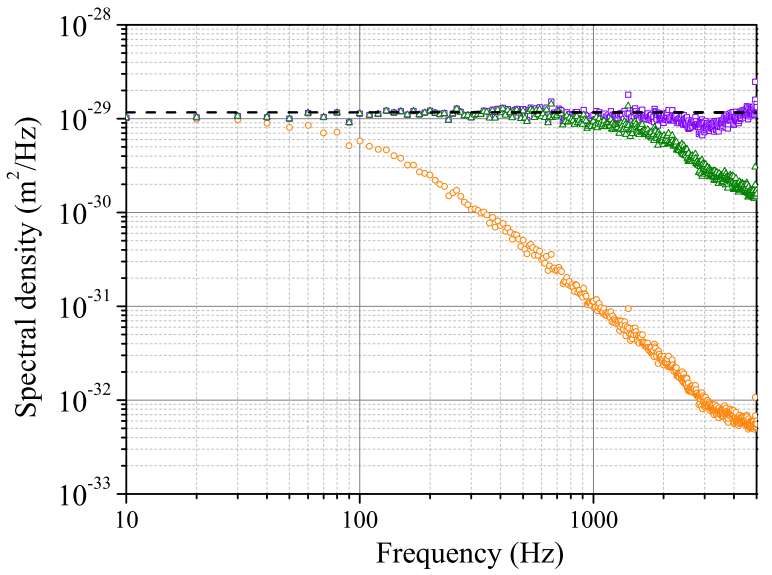

FIG. 2. (Color online) Measured spectral density in the $Y$ quadrature $\left(S_{Y m}\right)$ (orange circles), whitened spectrum (violet squares), with complete Wiener filtering (green triangles).

optomechanical resonance frequency at $\omega_{\mathrm{eff}}=\omega_{\mathrm{e}}$ and, as a consequence, its width $\gamma_{\text {eff }}$. Without control, optimal filtering would require an adaptive tuning of the parameters, that we are not trying to apply in this work.

\section{EXPERIMENT}

\section{A. Experimental apparatus}

A sketch of our experimental system is shown in Fig. 1(c). A Fabry-Perot cavity is formed between a micro-oscillator with high reflectivity dielectric coating as end mirror and a standard concave input coupler. The cavity length is $0.57 \mathrm{~mm}$, and its finesse is 57000 (half-line width $\kappa / 2 \pi=2.3 \mathrm{MHz}$ ). The input coupler is glued on a piezo-electric transducer for coarse tuning, and the cavity is kept in a vacuum chamber at $10^{-3} \mathrm{~Pa}$. The low-deformation micromirror $[19,20]$ has resonance frequency $\omega_{\mathrm{m}} / 2 \pi=128960 \mathrm{~Hz}$, mechanical quality factor $Q=\omega_{\mathrm{m}} / \gamma_{\mathrm{m}}=16000$ (limited, at room temperature, by thermoelastic losses), and effective mass $M=1.3510^{-7} \mathrm{~kg}$. More details on the measurements of the optomechanical parameters are reported in Refs. [19,21].

Two laser beams derived from the same Nd:YAG source are overlapped with orthogonal polarizations and optically matched to a cavity longitudinal mode with an efficiency of $\approx 96 \%$. From the reflected first beam (signal beam, with a power of $80^{\sim} \mu \mathrm{W}$ ) we obtain a dispersive profile of the optical resonance ( $\mathrm{PDH}$ signal) through phase modulation at 13.3 MHz and phase-sensitive detection [22]. Such a signal is exploited for locking the laser beam to the cavity resonance. Moreover, in the approximately linear region around resonance, the $\mathrm{PDH}$ signal is proportional to the oscillator displacement and is used both for monitoring its motion and in the parametric control loop described below. We remark that the bandwidth of the laser locking is kept at $\sim 30 \mathrm{kHz}$ (well below the mechanical frequency), and additional strong notch filters ensure that the laser frequency servo loop has no effect in the frequency region of interest (around the mechanical resonance).

The second beam (control beam), with a power of $1 \mathrm{~mW}$ at the cavity input, is frequency shifted with respect to the signal beam and is used to control the optical spring. The adjustable frequency shift compensates the cavity birefringence and determines the detuning of the control beam with respect to the cavity resonance. The ratio between optomechanical frequency shift due to the optical spring and control beam detuning is $8 \times 10^{-3} \mathrm{~Hz} / \mathrm{Hz}$. In addition, an electro-optic intensity modulator imposes a weak sinusoidal modulation in the power of the control beam and consequently in the radiation pressure acting on the micromirror.

The PDH signal is calibrated by means of a modulation at $\sim 20 \mathrm{kHz}$ sent to the laser frequency controller. The amplitude of this modulation at the input of the controller is directly measured during the acquisitions (since it is influenced by the frequency servo loop). This measurement as well as the measurement of the amplitude of the corresponding modulation in the PDH signal are repeated every $1 \mathrm{~s}$ during the data acquisition, in order to compensate for (weak) changes in the detection efficiency. The laser tuning rate (in $\mathrm{Hz} / \mathrm{V}$ ) had been previously calibrated with a Michelson interferometer, and the ratio between the laser frequency and the cavity length allows us to convert the detuning into cavity displacement. The overall calibration has an absolute accuracy of $\sim 20 \%$ (we point out that such uncertainty in the calibration factor does not influence the possibility to resolve weak signal variations, which is the object of this work).

The PDH signal is also sent to a double-phase, digital lock-in amplifier and integrated with a time constant of $80 \mu \mathrm{s}$. For the configuration with parametric control of the optomechanical frequency, the lock-in oscillator is sent to the intensity modulator of the control beam. A preliminary scan of its frequency allows us to reconstruct the response function of the mechanical oscillator and to tune the phase of the lock-in amplifier in order to have the dispersive component at the $X$ output. The reference oscillator is then set to $127400 \mathrm{~Hz}$, and the $X$ output of the lock-in amplifier is integrated and sent to the drivers of the acousto-optic modulators that vary the detuning of the control beam. The optomechanical resonance is now phase locked to the reference oscillator. The detuning of the control beam corresponds to about $0.09 \kappa$, and the oscillator is in a rather strong optical damping condition, with a resonance width of $\gamma_{\text {eff }} / 2 \pi \simeq 200 \mathrm{~Hz}$. For the configuration without parametric control, the effective optomechanical frequency is moved to about $127400 \mathrm{~Hz}$ by hand tuning the control beam, but the lock-in reference frequency is set at $127200 \mathrm{~Hz}$, so that the well-defined resonance peak at $\sim 200 \mathrm{~Hz}$ allows us to measure more accurately its parameters.

\section{B. Measurements}

The signal from the $Y$ output of the lock-in amplifier is acquired by a digital scope with a resolution of 12 bit and a sampling interval of $21 \mu \mathrm{s}$. Data are acquired by the scope in 35 consecutive time traces, each one lasting about $20 \mathrm{~s}$ (corresponding to $\sim 10^{6}$ data points) covering in all nearly $12 \mathrm{~min}$, then stored in a hard disk. Several such series are taken separated by periods of a few minutes (necessary to write the data on disk), for a total observation time of several tens of minutes.

The time series are divided into $100 \mathrm{~ms}$ long segments, a duration much larger than their correlation time. For 


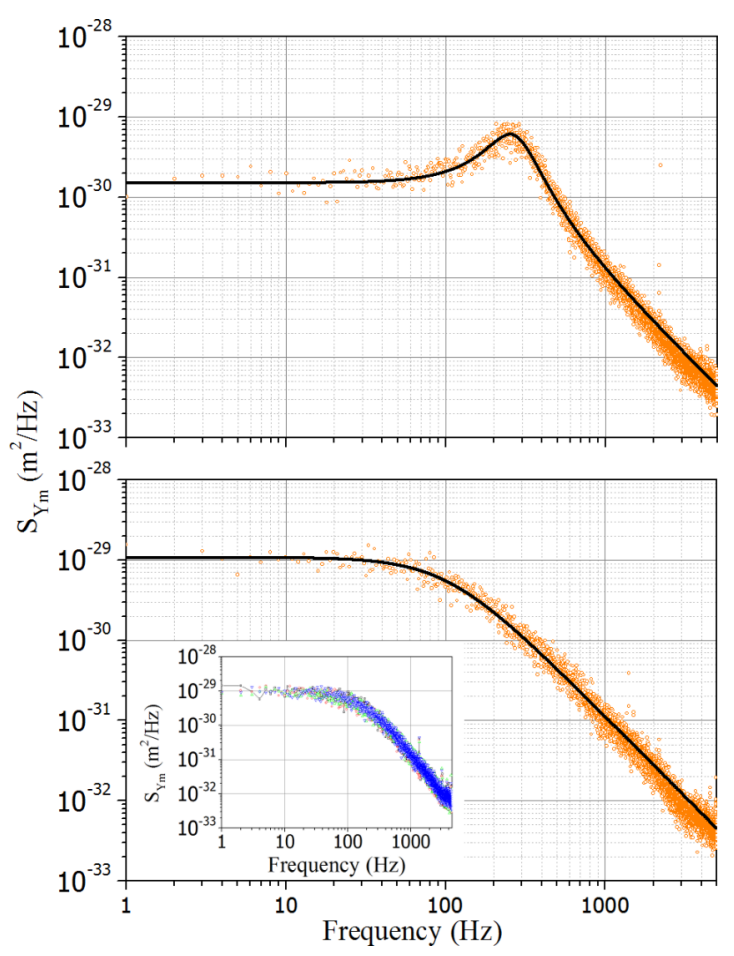

FIG. 3. (Color online) Spectral densities of the $Y$ quadrature $\left(S_{Y m}\right)$, for an oscillator without (upper panel) and with (lower panel) parametric control. With a solid line we show the respective fitting functions. In the inset, we compare spectra obtained with different values of the parametric control gain, showing that the control does not influence the dynamics of the $Y$ quadrature.

each section the power spectrum is calculated using a FFT algorithm, and corrected for the transfer function of the lock-in amplifier. The spectra corresponding to the first $20 \mathrm{~s}$ are averaged, and the averaged spectrum is fitted to Eq. (13) (when the parametric control is active) or to Eq. (15) (without control). An example of the averaged spectra and the fits are shown in Fig. 3. From the fitting procedure we obtain the resonance width, the signal maximum, and, in the absence of the control, the resonance frequency. The signal maximum Max is just exploited to define the value of the parameter $\Gamma$ to be used for Wiener filtering. For this purpose, we consider a conservative value of the background additive noise on $Y$, at $S_{B G}=8 \times 10^{-33} \mathrm{~m}^{2} / \mathrm{Hz}$ (one order of magnitude larger than the real $S_{n}$ ) and define $\Gamma=S_{B G} / \operatorname{Max}$. A typical value of $\Gamma$ is $10^{-3}$.

From each of the following spectra (after the first $20 \mathrm{~s}$ ) we calculate the force spectral density $S_{F}$ using the different methods described in the previous section (i.e., from the peak area and using Wiener filtering, both in the configuration with parametric feedback and with free-running oscillator). We report in Fig. 4 the average $\bar{S}_{F}\left(t_{\text {meas }}\right)$ of $S_{F}$ accumulated over $m$ consecutive spectra, corresponding to a measurement time $t_{\text {meas }}=m \tau$, where $\tau=100 \mathrm{~ms}$ is the time interval used for calculating each spectrum. The relative standard error is given by $\sigma_{\mathrm{REL}} \simeq 2 / \sqrt{t_{\text {meas }} \gamma_{\mathrm{eff}}}$ for the measurement with the peak area, and $\sigma_{\mathrm{REL}} \simeq \sqrt{2 \pi / t_{\text {meas }} \omega_{\text {cut }}}$ when using Wiener filtered data (these expressions refer to the configuration with parametric control where the peak is centered at null frequency,

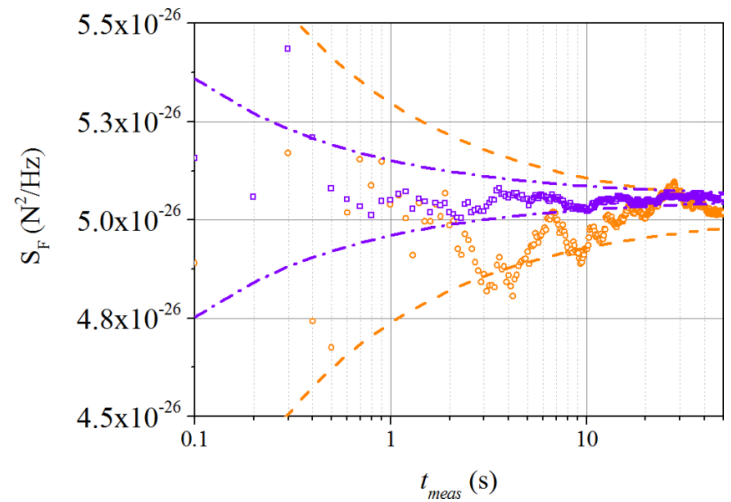

FIG. 4. (Color online) Average over a measurement time $t_{\text {meas }}$ of the force noise spectral density $S_{F}$, measured on the oscillator with parametric control using the peak area (orange circles) and the Wiener filtered spectra (violet squares). The confidence bands (respectively dashed and dash-dotted lines), corresponding to one standard error, are calculated in the Appendix.

and the latter relation is valid for $\omega_{\text {cut }} \ll \gamma_{\text {eff }} / 2 \sqrt{\Gamma}$; exact calculations are reported in the Appendix). $\sigma_{\mathrm{REL}}$ is used to calculate the confidence regions $\left(1 \pm \sigma_{\mathrm{REL}}\right) \bar{S}_{F}$, where $\bar{S}_{F}$ is the average at the end of the measurement period. The figure shows the expected convergence of the measured $\bar{S}_{F}\left(t_{\text {meas }}\right)$, which is clearly faster for the filtered data.

The calculation of the confidence region reported in Fig. 4 is just valid for a stationary system. A more reliable assessment on the measurement stability in the long term and on the achievable resolution is provided by the Allan variance [23]. In our case, its estimator is defined as

$$
\begin{aligned}
& \sigma_{A}^{2}(m)= \frac{1}{N-2 m+1} \sum_{k=1}^{N-2 m+1} \frac{\left(\bar{x}_{k+m}-\bar{x}_{k}\right)^{2}}{2}, \\
& \bar{x}_{k}(m)=\frac{1}{m} \sum_{n=k}^{k+m-1} S_{F}(n)
\end{aligned}
$$

where $S_{F}(n)$ is the value of force spectral density calculated from the $n$th spectrum and $N$ is the total number of spectra. The Allan deviation $\sigma_{A}(m)$ estimates the $1 \sigma$ uncertainty that can be obtained with a measurement lasting $t_{\text {meas }}=m \tau$. The calculated relative Allan deviation (i.e., $\sigma_{A}$ divided by $\bar{S}_{F}$ ) is reported in Fig. 5 for the different measurement strategies. We can derive two main considerations: (1) The measurement with Wiener filtering improves the statistical uncertainty much faster than the measurement from the peak area. For the former, a $1 \%$ resolution is obtained after $10 \mathrm{~s}$ and the best resolution of $0.4 \%$ is achieved, due to the parametric stabilization, after $1 \mathrm{~min}$, for the latter, the necessary measurement periods are about three times longer, in agreement with the ratio between the respective $\sigma_{\text {REL }}$. (2) For measurement periods exceeding $1 \mathrm{~s}$, the parametric control is crucial for the application of Wiener filtering. The measurement resolution does not improves any more after $1 \mathrm{~min}$ : with the parametric control it remains constant, while it becomes even worse without a control. It means that the parametric control also allows a much more relaxed choice of the optimal measurement time. 


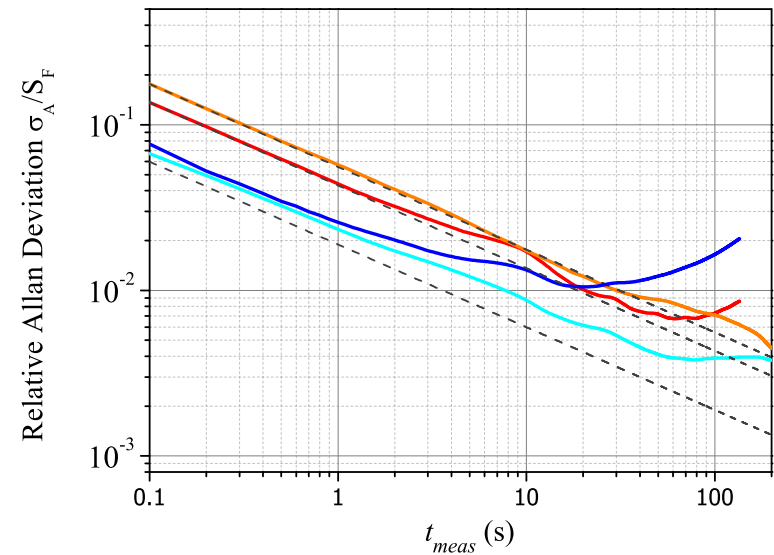

FIG. 5. (Color online) Relative Allan deviation concerning the measurement of the input stochastic force $S_{F}$, performed with four different procedures. Solid lines, from the upper to the lower curve (as seen in the left region of the graph): measurement from the peak area, with parametric control (orange); the same, without control (red); measurement from the Wiener-filtered data, without parametric control (deep blue); the same, with control (light blue). Dashed lines display the expected behavior in the absence of long-term effects, given by Eq. (A3) (upper line), Eq. (A8) (middle line), and Eq. (A5) (considering an implementation of the optimal filter; lower line).

\section{CONCLUSIONS}

We have analyzed different possible procedures for measuring the stochastic force acting on a micro-optomechanical system. In particular, we have compared the usual strategy based on the direct measurement of the area of the resonance peak (or, equivalently, of the variance in the oscillator displacement) with a more refined data analysis that approaches the optimal Wiener filtering. For the latter case, we have introduced an abrupt bandwidth limitation that allows a near-optimal realistic measurement procedure. We have shown that, while for the former method, the optical damping, decreasing the oscillator coherence time can improve the resolution of the measurement in a given observation time, the appropriate filtering gives sensibly better results, which are mostly independent on such coherence time.

The implementation of the Wiener filtering is greatly facilitated and more effective by using a parametric control of the oscillator frequency, a technique that we have recently introduced and that we have analyzed here in detail. Due to such active stabilization, our system can reliably detect variations of the stochastic force below $1 \%$ within $1 \mathrm{~min}$. We remark that a correct assessment of the really achievable resolution with long integration periods cannot be simply based on the convergence of the averaged measurement. Indeed, such indicator underestimates the effect of system long-term instabilities and parameter drifts. Using the Allan variance as the correct estimator, we show that parametric control plays a crucial role in the achieved performance.

The procedure for the measurement of the stochastic force that we have described in this work, including optimal filtering and parametric control, can be applied in a large variety of micro- and nano-mechanical systems, including those based on electric measurements and microwave radiation. Detecting a weak stochastic signal on a stronger background is an important task in the research field of quantum mechanics with macroscopic oscillators, in particular when exploring the properties of oscillators with low occupation number, or, e.g., in a squeezed state [16,24-26] or other peculiarly quantum states. In this situation, the measurement back-action can destroy the interesting features. Particular measurement schemes can be conceived and applied [24,25,27,28], but the use of a weak measurement, where the signature of the oscillator is intrinsically weaker than the measurement noise (see, e.g., Ref. [29]), can be a useful affordable solution. The procedures investigated in this work would thus provide valuable help.

\section{ACKNOWLEDGMENTS}

F.M. thanks M. Prevedelli for the discussion on phase locking. This work has been supported by the European Commission (ITN-Marie Curie project cQOM), by MIUR (PRIN 2010-2011), and by INFN (HUMOR project).

\section{APPENDIX}

We consider a Gaussian, zero mean stochastic process $x(t)$ with finite variance $\sigma_{x}^{2}$, correlation function $C_{x x}$, and power spectral density $S_{x x}$. The estimate of the mean square of $x(t)$ in the interval $\left[0, t_{\text {meas }}\right]$ has expectation value $\sigma_{x}^{2}$ and standard deviation $[10,30]$

$$
S T D \simeq\left[\frac{2}{t_{\text {meas }}} \int_{-\infty}^{\infty} C_{x x}^{2}(\tau) d \tau\right]^{\frac{1}{2}}
$$

The relative standard deviation is defined as $\sigma_{\mathrm{REL}}=S T D / \sigma_{x}^{2}$, and it can be expressed in terms of spectral densities using

$$
\begin{aligned}
\sigma_{x}^{2} & =\int_{-\infty}^{\infty} S_{x x}(\omega) \frac{d \omega}{2 \pi}, \\
S T D & \simeq\left[\frac{2}{t_{\text {meas }}} \int_{-\infty}^{\infty} S_{x x}^{2}(\omega) \frac{d \omega}{2 \pi}\right]^{\frac{1}{2}} .
\end{aligned}
$$

For a spectrum given by $S_{x x}(\omega) \propto \mathcal{L}(\omega)$ we obtain the relative standard deviation [10]

$$
\sigma_{\mathrm{REL}}=\frac{2}{\sqrt{t_{\text {meas }} \gamma_{\mathrm{eff}}}} .
$$

This expression can be used for the relative uncertainty in the measurement of $S_{F}$ using the peak area, since in this case we can neglect the measurement noise $S_{n}$ and the finite integration band defined by $\omega_{\text {cut }}$. For the Wiener-filtered process, using Eqs. (13) and (16) and the expression of $S_{W}$ and $\Gamma_{\text {opt }}$ we can write the output spectrum in the form

$$
S_{x x} \propto \mathcal{L}(\omega) \frac{\mathcal{L}(\omega)+\mathcal{L}(0) \Gamma_{\mathrm{opt}}}{[\mathcal{L}(\omega)+\mathcal{L}(0) \Gamma]^{2}}
$$




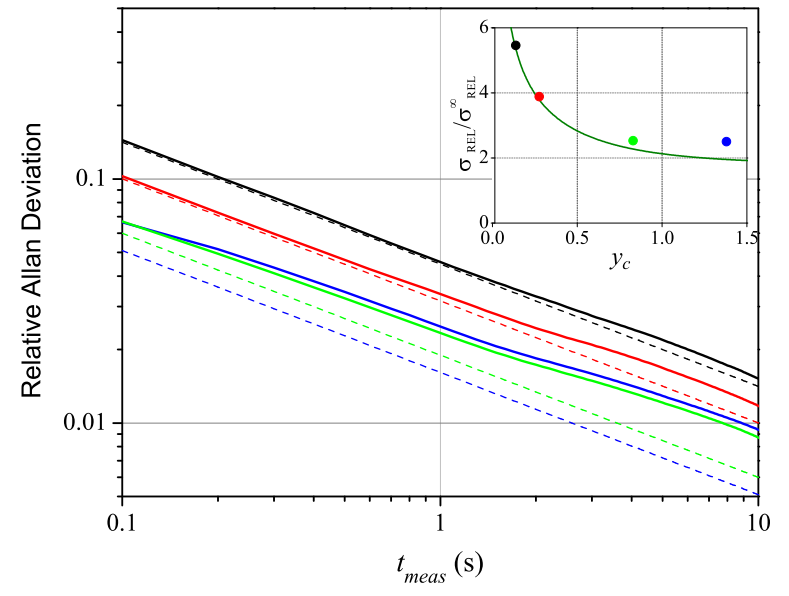

FIG. 6. (Color online) Relative Allan deviation for the measurement of $S_{F}$ using the Wiener filtered data, for different values of the cutoff frequency. The dashed lines correspond to the calculated $\sigma_{\text {REL }}$ for $\omega_{\text {cut }} / 2 \pi=500 \mathrm{~Hz}, 1 \mathrm{kHz}, 3 \mathrm{kHz}$, and $5 \mathrm{kHz}$ (from the upper to the lower line). The solid curves are the experimental results, and we see that the data extracted with the cutoff at $5 \mathrm{kHz}$ overtake the curve corresponding to $\omega_{\text {cut }} / 2 \pi=3 \mathrm{kHz}$. In the inset, the experimental relative Allan deviation at $t_{\text {meas }}=0.1 \mathrm{~s}$, normalized to the corresponding $\sigma_{\mathrm{REL}}^{\infty}$, is reported for the same values of $\omega_{\text {cut }}$ and compared with the theoretical behavior [calculated from Eqs. (A5)-(A6)] shown with a solid line.

and the relative standard deviation as

$$
\begin{aligned}
\sigma_{\mathrm{REL}} & =\frac{2}{\sqrt{t_{\text {meas }} \gamma_{\mathrm{eff}}}}\left(\frac{\Gamma}{1+\Gamma}\right)^{\frac{1}{4}} \frac{\sqrt{\pi \int_{0}^{y_{c}\left[\frac{1+g y^{2}}{\left(1+y^{2}\right)^{2}}\right]^{2} d y}}}{\int_{0}^{y_{c}} \frac{1+g y^{2}}{\left(1+y^{2}\right)^{2}} d y}, \\
y_{c} & =\omega_{\mathrm{cut}} \frac{2}{\gamma_{\mathrm{eff}}} \sqrt{\frac{\Gamma}{1+\Gamma}}, \\
g & =\frac{\Gamma_{\mathrm{opt}}(1+\Gamma)}{\Gamma\left(1+\Gamma_{\mathrm{opt}}\right)} .
\end{aligned}
$$

It is useful to consider the two limits $y_{c} \rightarrow \infty$ and $y_{c} \ll 1$, that for $g \ll 1$ and $\Gamma \ll 1$ can be written, respectively, as

$$
\sigma_{\mathrm{REL}}^{\infty} \simeq \sqrt{\frac{10}{t_{\text {meas }} \gamma_{\mathrm{eff}} / \sqrt{\Gamma}}}
$$

and

$$
\sigma_{\mathrm{REL}} \simeq \sqrt{\frac{2 \pi}{t_{\text {meas }} \omega_{\mathrm{cut}}}} .
$$

In the inset of Fig. 6 (solid line) we show the behavior of $\sigma_{\mathrm{REL}} / \sigma_{\mathrm{REL}}^{\infty}$ as a function of $y_{c}$. The relative accuracy is just $20 \%$ worse if the integration is limited to $y_{c}=1$, but, on the other hand, a frequency cutoff allows us to reject spurious signals that can appear around the interesting resonance.

For a spectrum formed by a couple of symmetric Lorentzian peaks centered at $\pm \delta \omega$ [see Eq. (15)], the relative standard deviation, when measuring directly the peaks area, becomes

$$
\sigma_{\mathrm{REL}}=\frac{2}{\sqrt{t_{\text {meas }} \gamma_{\mathrm{eff}}}} \sqrt{\frac{\gamma_{\mathrm{eff}}^{2}+2 \delta \omega^{2}}{\gamma_{\mathrm{eff}}^{2}+4 \delta \omega^{2}}} .
$$

The Wiener filter is obtained from the expression for a single peak, given in Eq. (16), by replacing $\mathcal{L}(\omega) \rightarrow 0.5[\mathcal{L}(\omega-$ $\delta \omega)+\mathcal{L}(\omega+\delta \omega)]$. Due to the spectral flattening action of the Wiener filter, the filtered output is very similar to the case of the single peak. As a consequence, for our typical parameters, the two theoretical values of $\sigma_{\text {REL }}$ differ by less than $1 \%$.

The relative Allan deviation is equal to $\sigma_{\mathrm{REL}}$ in the absence of excess fluctuations (typically, for short measurement time). In our experiment, $y_{c}=1$ for $\omega_{\text {cut }} / 2 \pi \simeq 6300 \mathrm{~Hz}$. In Fig. 6 we report the measured relative Allan deviation as a function of $t_{\text {meas }}$ for different values of the cutoff frequency, together with its expected behavior. When $\omega_{\text {cut }} / 2 \pi$ surpasses $3 \mathrm{kHz}$, the presence of additional peaks starts to influence the measurement. This is also visible in the inset, where the measured relative Allan deviation at $t_{\text {meas }}=0.1 \mathrm{~s}$, normalized to the calculated $\sigma_{\mathrm{REL}}^{\infty}$, is reported for different values of $\omega_{\text {cut }}$ and compared with the theoretical behavior.
[1] E. Gavartin, P. Verlot, and T. J. Kippenberg, Nat. Nanotech. 7, 509 (2012).

[2] H. Miao, K. Srinivasan, and V. Aksyuk, New J. Phys. 14, 075015 (2012).

[3] M. Poggio, Nat. Nanotech. 8, 482 (2013).

[4] T. P. Purdy, R. W. Peterson, and C. A. Regal, Science 339, 801 (2013).

[5] D. Kleckner and D. Bouwmeester, Nature (London) 444, 75 (2006).

[6] S. Gigan et al., Nature (London) 444, 67 (2006).

[7] O. Arcizet et al., Nature (London) 444, 71 (2006).

[8] A. N. Kolmogorov, Bull. Acad. Sci. USSR Scr. Math. 5, 3 (1941).

[9] N. Wiener, Extrapolation, Interpolation, and Smoothing of Stationary Time Series: With Engineering Applications (MIT Press, Cambridge, MA; Wiley and Sons, New York; Chapman and Hall, London, 1949).
[10] P. Astone, P. Bonifazi, and G. V. Pallottino, Rev. Sci. Instrum. 61, 3899 (1990).

[11] J. Tamayo, J. Appl. Phys. 97, 044903 (2005).

[12] A. Vinante, M. Bonaldi, F. Marin, and J.-P. Zendri, Nat. Nanotech. 8, 470 (2013).

[13] G. I. Harris, D. L. McAuslan, T. M. Stace, A. C. Doherty, and W. P. Bowen, Phys. Rev. Lett. 111, 103603 (2013).

[14] D. Antonio, D. H. Zanette, and D. Lopez, Nat. Commun. 3, 806 (2012).

[15] E. Gavartin, P. Verlot, and T. J. Kippenberg, Nat. Commun. 4, 2860 (2013).

[16] A. Pontin, M. Bonaldi, A. Borrielli, F. S. Cataliotti, F. Marino, G. A. Prodi, E. Serra, and F. Marin, Phys. Rev. Lett. 112, 023601 (2014).

[17] C. Genes, D. Vitali, P. Tombesi, S. Gigan, and M. Aspelmeyer, Phys. Rev. A 77, 033804 (2008). 
[18] V. B. Braginsky, M. L. Gorodetsky and F. Ya. Khalili, Phys. Lett. A 232, 340 (1997).

[19] E. Serra, A. Borrielli, F. S. Cataliotti, F. Marin, F. Marino, A. Pontin, G. A. Prodi, and M. Bonaldi, Appl. Phys. Lett. 101, 071101 (2012).

[20] E. Serra, A. Bagolini, A. Borrielli, M. Boscardin, F. S. Cataliotti, F. Marin, F. Marino, A. Pontin, G. A. Prodi, M. Vannoni, and M. Bonaldi, J. Micromech. Microeng. 23, 085010 (2013).

[21] E. Serra, A. Borrielli, F. S. Cataliotti, F. Marin, F. Marino, A. Pontin, G. A. Prodi, and M. Bonaldi, Phys. Rev. A 86, 051801 (2012).

[22] R. W. P. Drever et al., Appl. Phys. B 31, 97 (1983).
[23] D. W. Allan, Proc. IEEE 54, 221 (1966).

[24] A. A. Clerk, F. Marquardt, and K. Jacobs, New J. Phys. 10, 095010 (2008).

[25] J. B. Hertzberg, T. Rocheleau, T. Ndukum, M. Savva, A. A. Clerk, and K. C. Schwab, Nat. Phys. 6, 213 (2009).

[26] A. Szorkovszky, G. A. Brawley, A. C. Doherty, and W. P. Bowen, Phys. Rev. Lett. 110, 184301 (2013).

[27] J. Chan et al., Nature (London) 478, 89 (2011).

[28] A. Kronwald, F. Marquardt, and A. A. Clerk, Phys. Rev. A 88, 063833 (2013).

[29] J. D. Thompson et al., Nature (London) 452, 72 (2008).

[30] J. S. Bendat and A. G. Piersol, Random Data: Analysis and Measurement Procedures (Wiley, New York, 2010). 